\title{
S35-01
}

\section{WHICH SYMPTOMS DO BEST PREDICT MAJOR DEPRESSIVE DISORDER (MDD) IN MALES? RESULTS FROM AN ICELANDIC COMMUNITY STUDY}

\author{
S. Pálsson ${ }^{1}$, B. Sigurðsson², Ó. Aevarsson ${ }^{3}$, M. Ólafsdóttir ${ }^{4}$ \\ ${ }^{1}$ Department of Psychiatry, University Hospital, Landspitali, ${ }^{2}$ Department of Pharmacology and Toxicology, University of Iceland, \\ ${ }^{3}$ Forvarnier EHF, ${ }^{4}$ Faculty of Medicine, University University of Iceland, Reykjavík, Iceland
}

Background/objectives: The concept of male depression is based on the hypothesis that typical symptoms of depression in men often seem to be masked by non-typical male distress symptoms not considered in common depression inventories. Despite clinical evidence, scientific evidence is still lacking. The study describes symptoms reported by males (on the Gotland Male Depression Scale (GMDS) in a community study and these were further validated against MDS criteria.

Methods: In a community study in Reykjanes Iceland, 534 males were rated by the GMDS. In the second phase a sub-sample of healthy and depressive males were examined by a trained psychiatrist in a semi-structured interview ( $N=137)$. Diagnosis was according to the DSM-IV for MDD. The symptoms on GMDS are self-rated from 0-3.

Results: In the community sample the prevalence (>1 GMDS) of single symptoms was following: More stressed out than usual $(16.8 \%)$, aggressiveness $(11.8 \%)$, feeling of being burned out $(14.5 \%)$, tiredness $(18.2 \%)$, more irritable $(15.3 \%)$, difficulty making decisions $(10.8 \%)$, sleep problems $(21 \%)$, anxiety/uneasiness (10.5\%), increased use of alcohol/substances, hyperactivity (10.3\%), behavioural changes $(4.7 \%)$, feeling sad/hopeless (10.4\%), self-pity (4.8\%). All symptoms on GMDS correlated strongly with MDD. In a stepwiselogistic regression model, controlling for medication and age, three items contributed independently to MDD diagnoses. These were aggressiveness, being more irritable and anxiety/uneasiness.

Conclusions: Sleep problems and tiredness were most commonly reported symptoms. The best predicting symptoms for MDD were anxiety and acting out symptoms, aggression and irritability. This is of interest as these symptoms are not in the criteria for MDD. 